\title{
Connection and Resource allocation of IoT Sensors to cellular technology- LTE
}

\author{
Darshana Thomas \\ Department of Electronic and \\ Electrical Engineering \\ Strathclyde University \\ Glasgow, G1 1XW \\ Email: dershana.thomas@strath.ac.uk
}

\author{
James Irvine \\ Department of Electronic and \\ Electrical Engineering \\ Strathclyde University \\ Glasgow, G1 1XW \\ Email: j.m.irvine@strath.ac.uk
}

\begin{abstract}
The number of sensor nodes being used for the applications is increasing dramatically every year. In this paper we consider LTE for sensor networks. There is little published research on resource allocation of sensor networks in LTE. Theoretical calculation carried out for mobile users in LTE was used and recreated for sensor nodes transmitting. Existing literature has carried this out for mobile users on LTE network, transmitting $10 \mathrm{~Kb}$, theoretically without mentioning the total resource blocks allocated for the network based on the bandwidth. In this paper we utilise LTE for sensor networks and investigate how many sensor nodes can transmit and how many bytes will be transmitted per Resource Block (RB). Theoretical calculation was carried out for sensor nodes to find out the data that can be transmitted per RB by sensor nodes transmitting $10 \mathrm{~Kb}$. We find significantly different results when carrying out simulation for a critical sensor network, using realistic real world parameters for the LTE network. We clearly specify the bandwidth and resource blocks used for the simulation in this paper. We carry out simulation in OMNET to create sensor network scenario demonstrating results with 29 sensor nodes transmitting $1 \mathrm{~Kb}$ of data.
\end{abstract}

Keywords: Sensors, Resource Allocation, LTE

\section{INTRODUCTION}

The past few years has seen an explosion in the availability of small, programmable, integrated wireless modules which enable small connected sensors [1]. These modules have developed from Bluetooth devices with relatively simple stacks to Wi-Fi modules such as the Texas Instruments CC3000 [2] , which for less than 10 dollar in low quantities includes a complete 802.11g subsystem, IP stack and web server, with only a few external capacitors and chip antenna required for a complete design. As modules become more powerful, the inclusion of an IP stack allows rapid development and enables the 'Internet of Things' concept, where sensors are fully connected IP nodes rather than reliant on bespoke wireless protocols. Sensor an M2M device needs to be upgraded to recent technologies. Sensors are devices that detect signals and respond to input. Output is gathered electronically for futher processing. According to recent cisco white paper [3] growth of machine to machine communication in everyday lives is increasing. Figure below shows the migration of M2M devices through $2 \mathrm{G}, 3 \mathrm{G}$ and $4 \mathrm{G}$.

In areas beyond Wi-Fi coverage, it is likely that the advantages of rapid development and ubiquity are going to

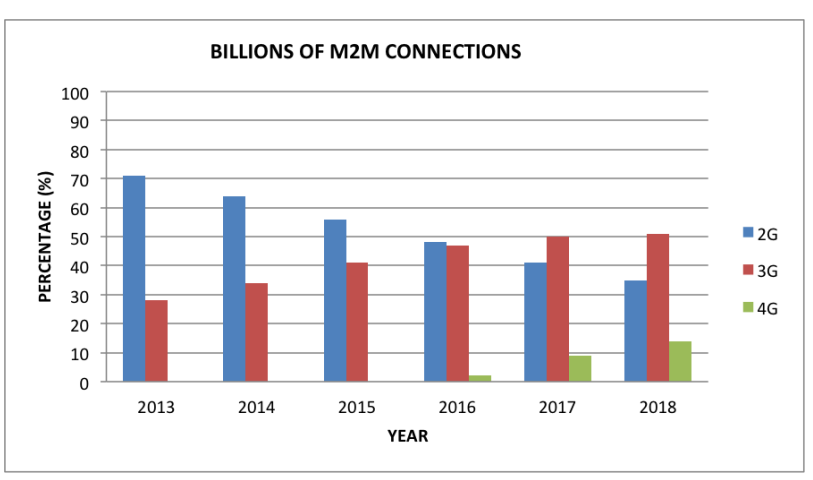

Fig. 1. M2M Connections

suggest the IP route as well. Currently cheap wireless modules are restricted to $2 \mathrm{G}$ GPRS technology, but the integration seen in other wireless technologies is likely to be applied here (albeit that licensing costs form a greater part of cellular technology that WLAN). With $4 \mathrm{G}$ currently being deployed, we anticipate that cellular modules for sensors will leapfrog $3 \mathrm{G}$ technology to apply 4G LTE technology, especially since LTE has a much more flexible resource management architecture and will be able to cope with large numbers of sensors more efficiently than previous technologies. LTE offers uplink of $50 \mathrm{Mbps}$, downlink speed of 100Mbps, low latency and high cell edge performance [4]. This paper therefore considers the practical capacity of LTE to support the sort of IoT sensors which are beginning to become available. Even though the capacity of LTE is well known, authors have not verified if the capacity can be utilised for sensor networks which will require very many transmissions with limited data. In this paper we utilise the capacity of LTE for sensor networks. Research such as [5], have been carried out on wireless sensor networks but very few researches are carried out on resource allocation of wireless sensors in LTE. Most of the research done is based on fixed packet size and periodic transmission from sensors but in many applications this will not be realistic. In the next section LTE architecture and resource allocation is explained followed by the case study for the paper, simulation and conclusion.

LTE architecture is divided into two levels: Evolved Packet Core-EPC (user plane low level) and Enhanced UMTS Terrestrial Radio Access Network-EUTRAN (control plane high level). Each level has different elements to it, which would 
combine together to make a complete LTE network architecture [6]. There are sets of protocol stacks for each element within the network architecture [7].

\section{A. Header Compression}

Like UMTS, LTE has a Packet Data Convergence Protocol (PDCP) entity in the upper part of layer 2 to more efficiently handle packet data. When IP packets are being transported, they are delivered from layer 3 to the PDCP, which performs IP header compression. The $20+$ byte IP header is replaced with a PDCP header of 1-4 bytes before the resulting PDU is passed to the RLC. RFC2507 is used for header compression, which requires the transmission of the full header periodically for context. An issue with header compression is that while it is effective for continuous data streams at reducing the amount of header data transmitted, where only a few packets are transmitted, such as for sensor nodes transmitting one or two IP packets, the savings will be much less. Therefore, the capacity predicted for IP data streams over LTE when sending large amounts of data will not directly scale when smaller amounts of data are being sent.

\section{RESOURCE AllocAtion in LTE}

Resource allocation in LTE is a key feature of this system enabling users to transmit and receive. Scheduling of resource allocation within an LTE network is carried out by base stations based upon the availability of these resources. There are two types of scheduling in LTE: uplink and downlink scheduling. In an LTE network, the base station controls scheduling. The advantages of a base station controlling scheduling are ensuring quality of service for each user, dealing with overload situations and optimizing overall throughput by reacting to changing radio conditions of each user. The base station would decide the number of resource blocks to be allocated to each user and how many users to be scheduled. Bandwidth slots allocated for LTE is between $1.4 \mathrm{MHz}$ and $20 \mathrm{MHz}$. The higher the bandwidth, the higher number of resource blocks available [8].

Figure 2, depicts LTE resource allocation done in two domains: time domain and frequency domain. In the time domain 10 sub frames are available where each frame represents 2 slots of $0.5 \mathrm{~ms}$ slot. Each $1 \mathrm{~ms}$ frame results in a TTI-time transmission interval- which are theoretically responsible to transmit. Each $0.5 \mathrm{~ms}$ slot has 1 resource block made of 12 subcarriers and 6 or 7 OFDM symbols. Even though resource allocation uses resource blocks it is actually the subcarriers within each resource block that contain uplink and downlink transmission. The allocated frequency for each resource block is $180 \mathrm{kHz}$ [9]. Each lost packet within a cell results in more retransmissions having to take place to redeliver the packets, which ends up in increased latency and high number of resources been used up, which could have been used to allocate more users if no retransmission occurred. Practically it is almost impossible to avoid retransmission even in an LTE network, as many factors within each cell served by a base station would be affected by many factors such as building and environment noise etc.

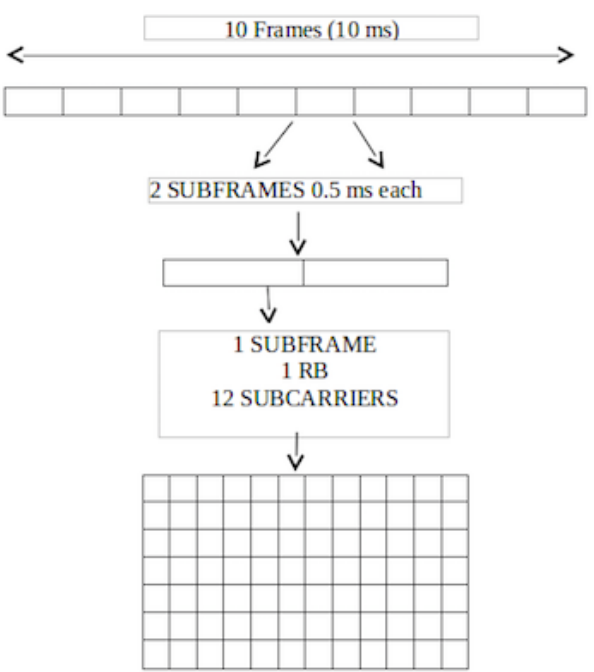

Fig. 2. LTE Frame Structure

\section{CASE STUdY}

We are using an uncoordinated number of sensor nodes to connect to LTE and push data across the network. In the scenarios created for the simulation section, sensor nodes are transmitting data across the network. LTE can theoretically

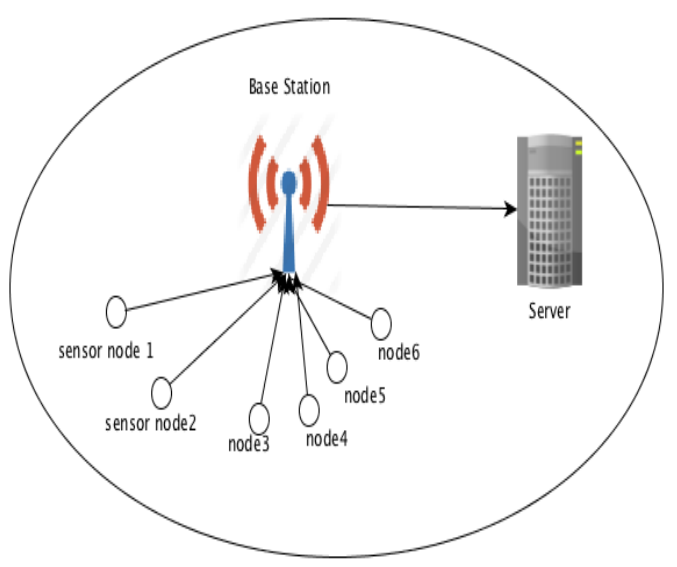

Fig. 3. LTE Sensor Scenario

allocate resources for many number of users within the network. We use the information from paper [10] to work out the number of sensor nodes that can transmit the same amount of data with 55 resource blocks. The simulation set up will be as close to real world situations where sensor nodes would send feedback to base station. Figure 3 shows the scenario for sensor nodes communicating to a base station to transmit. We assume for this paper that the sensor node has enough power to transmit.

The parameters used in the paper [10] are:

$$
\begin{gathered}
\mathrm{CQI} \leftarrow 14 \\
\mathrm{Bit} / \mathrm{Hz} \leftarrow 5.1
\end{gathered}
$$

Data $\leftarrow 10 \mathrm{~Kb}$

Number of resource blocks used is 14 
14 resource blocks are utilized while transmitting $10 \mathrm{~Kb}$ of data. In paper [10] the bandwidth or the number of total resource blocks allocated for transmission is not stated instead the resource block utilised is stated. In this paper we clearly state the bandwidth used and the total resource blocks that can be used for transmission. We use the same amount of data for sensor nodes to know if it is possible to transmit $10 \mathrm{~Kb}$ of data with the allocated resource blocks realistically based on results obtained through simulation. If the same calculation was carried out where the network is allocated $10 \mathrm{MHz}$ bandwidth, 55 resource blocks and $180 \mathrm{kHz}$ for each resource block. The calculation would be based upon parameters described below:

$$
\begin{gathered}
\mathrm{CQI} \leftarrow 14 \\
\mathrm{kHz} \leftarrow 180 \\
\text { Data } \leftarrow 10 \mathrm{~Kb} \\
\text { Bandwidth } \leftarrow 10 \mathrm{MHz}
\end{gathered}
$$

If we assume that all $10 \mathrm{~Kb}$ of data was allowed to be transmitted by the sensor nodes at once. The calculation will work out to be 181 bytes transferred per RB as there are only 55 RBs. The simulation carried out in the next section would allow us to ascertain if it is possible to transmit a total of $10 \mathrm{~Kb}$ of data with 181 bytes per RB.

\section{DATA MODEL}

The above theoretical results assume constant data rates of $10 \mathrm{~Kb} / \mathrm{s}$. Sensor nodes will transmit data intermittently.

Sensors are likely to transmit very small amounts of data. For example, a temperature sensor may only have to transmit a single temperature value, which could be encoded as a single byte. Following the examples at [11], and assuming a Spark.IO device is a typical internet connected sensor, we find that a minimal set of headers for the HTTP request is in the order of 100 characters, and a minimal body is about 50 characters. However, a more normal (and secure) 'small' packet is in the order of 250 bytes, and with large packets reporting a number of measurements approaching 1000 bytes. We have therefore used these values for our simulations.

\section{Simulation}

In order to accurately model LTE, we used the SimuLTE [12] extension for Omnet [13]. SimuLTE accurately models LTE down to the physical layer, including the PDCP, RLC and MAC, status and channel reporting, etc. Physical transmission at a symbol level over OFDM is abstracted [13], but the model implements a channel model which allows us to predict whether packets will be corrupted and require retransmission. The scenario created was a simple single cell with sensors distributed over a $300 \mathrm{~m}$ by $300 \mathrm{~m}$ square area. Since we are interested in the relative requirement for resource blocks, we did not consider retransmissions at the application layer if a packet timed out at the RLC it was considered dropped. Sensors transmitted at random intervals over the course of a second, and all transmissions were completely within that time. Two configurations were considered sensors transmitting 250 bytes and sensors transmitting 1000 bytes.

Simulation was run several times to gather results with 95 percent confidence interval.

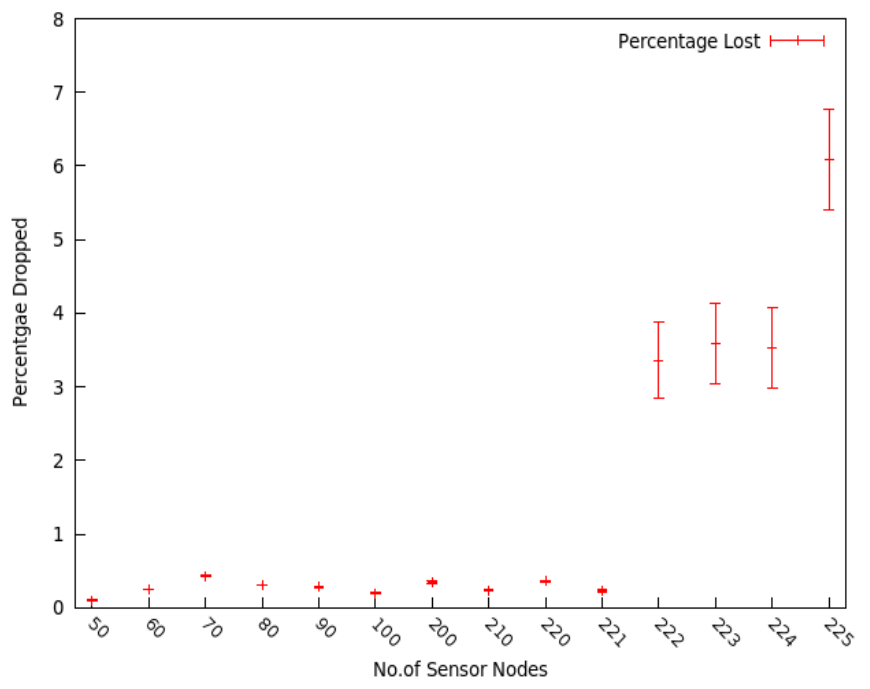

Fig. 4. Percentage Dropped for nodes transmitting at 300m (250 bytes each)

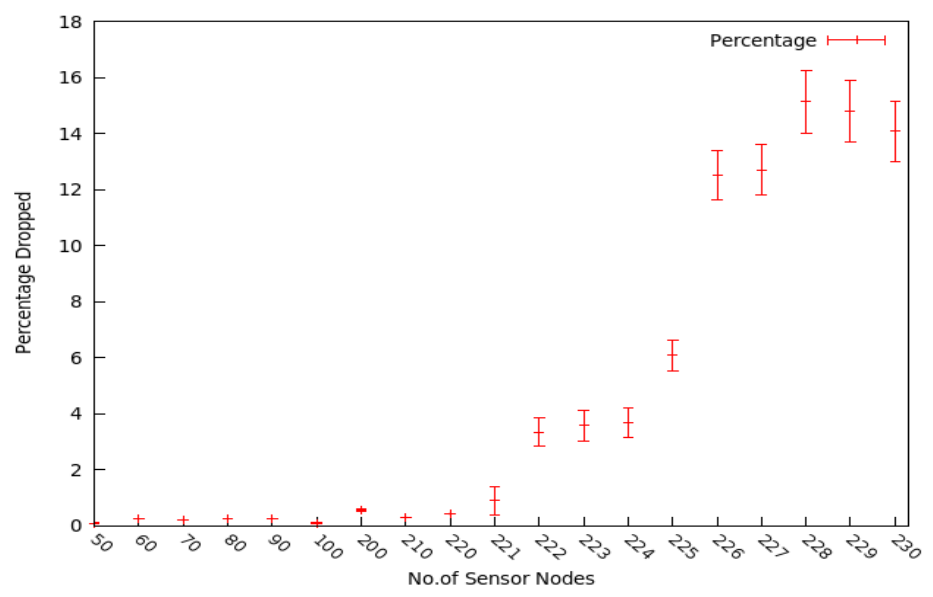

Fig. 5. Percentage Dropped for nodes transmitting at $800 \mathrm{~m}$ (250 bytes each)

Figure 4 represents percentage dropped for results obtained for transmission of data by the sensor nodes at $300 \mathrm{~m}$. Each node transmitted 250 bytes each. The bandwidth allocated for the system is $10 \mathrm{MHz}$. From Figure 4 it can be noted that 221 IoT sensor nodes could transmit with almost zero percent delay. Anything above 221 nodes transmitting, started losing packets which has resulted in very high percentage drop. Figure 5 was the exact same setting as the other one but this time distance was increased to $800 \mathrm{~m}$ to ensure the scenario is realistic as possible and if it is then a propagation delay was expected. As expected, in Figure 5 it can be seen that there is a propagation delay which resulted in loss of packets for 221 nodes transmitting whereas for $300 \mathrm{~m}$ transmission 221 nodes transmitted with no packet loss. The next set of results Figure 6 and Figure 7 was results obtained for nodes transmitting 1000 bytes at $300 \mathrm{~m}$ and $800 \mathrm{~m}$.

The percentage drop results are presented with an upper limit and lower limit based on 95 percent confidence interval. As the number of sensor nodes decreases there is a significant amount of decrease in percentage drop of lost packets. 29 sensor nodes transmitted 2302 bytes with less than one percent 


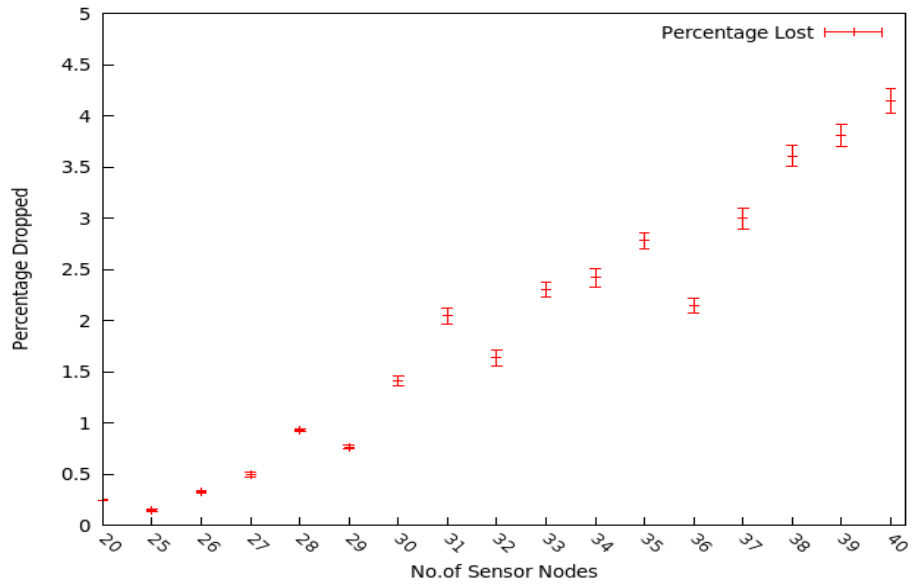

Fig. 6. Percentage Dropped for nodes transmitting at 300m (1000 bytes each)

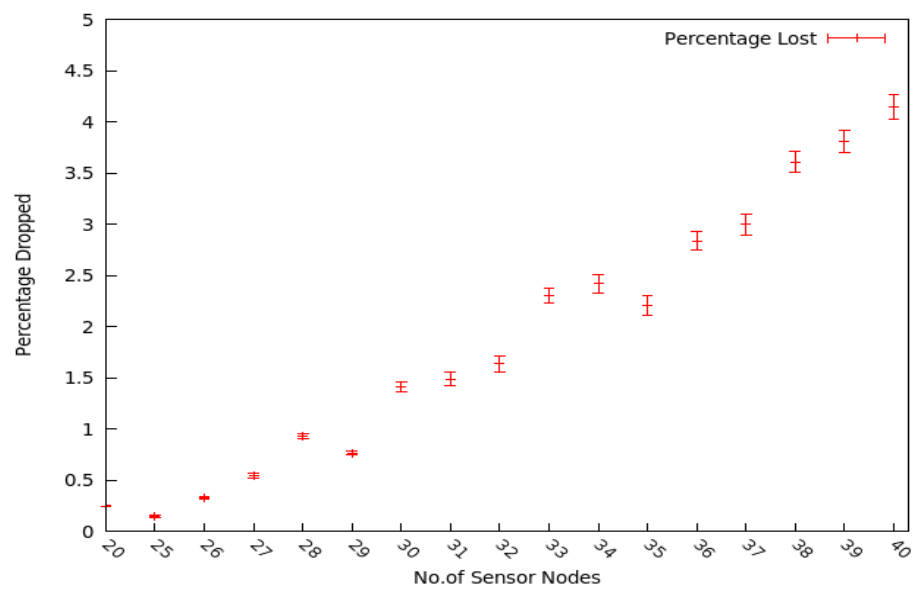

Fig. 7. Percentage Dropped for nodes transmitting at $800 \mathrm{~m}$ (1000 bytes each)

packet drop. Figure 4 and Figure 5 shows the results obtained for sensors transmitting 250 bytes. 100 sensors transmitted with less than 1 percent packet loss from Figure 4.

Following the results stated in Section III, we estimated the number of users transmitting $10 \mathrm{~Kb}$ of data each second, and found that such a system could support 80 users. This corresponds to a useful rate of some 181 bytes per resource block. However, when transmitting to individual users discontinuously in full simulation with resource allocation, etc, less than 29 users transmitting 1000 bytes could be supported in each second, corresponding to a usable rate of 21 bytes per resource block, a very significant reduction if only 1 percent packet dropping is required (admittedly a tight constraint for something like sensor reporting).

These results assume that the sensors transmit infrequently enough that resources are not allocated to them between sessions, but this is likely if the sensors are transmitting only a few times each hour or less.

\section{CONCLUSION}

LTE was utilised for sensor nodes to find out how effective it is for sensor nodes in terms of resource allocation. The packet size for the sensor nodes was chosen based on current trends in IoT sensors being deployed now. Existing work on mobile users in an LTE network has calculated theoretical numbers of RBs needed to carry $10 \mathrm{~Kb}$ of data continuously, but this significantly overestimates practical performance when sensors have to register with the system, request resource, and then transmit. In this paper the total resource blocks used for transmission are shown to be much higher than would be suggested by the theory. Simulation was carried out using SimuLTE in OMNET using real world parameters for sensor networks in an LTE network show that a maximum of $1 \mathrm{~Kb}$ data can be transmitted by 29 sensor nodes each second with less than 1 percent of packet loss which equates to 21 bytes of data being transmitted per RB, compared to 181 bytes per resource block for the continuous transmission.

\section{REFERENCES}

[1] Sarah Perez. The Number Of Mobile Devices Will Exceed Worlds Population By 2012, February 2012. http://techcrunch.com/2012/02/14/thenumber-of-mobile-devices-will-exceed-worlds-population-by-2012other-shocking-figures/.

[2] Texas Instruments. CC3000 IEEE $802.11 \mathrm{~b} / \mathrm{g}$ Solution Module. http://www.ti.com/product/cc3000.

[3] Cisco. Architectural Considerations for Backhaul of $2 \mathrm{G} / 3 \mathrm{G}$ and Long Term Evolution Networks.

[4] Farooq Khan. LTE for 4G Mobile Broadband. 2009.

[5] Sasan Adibi, Amin Mobasher, and Tom Tofigh. LTE networking: extending the reach for sensors in mHealth applications. Transactions on Emerging Telecommunications Technologies, 2013.

[6] Telesystem Innovations. LTE in a Nutshell. White Paper, 2010.

[7] Hyung G Myung. Technical overview of 3GPP LTE. Polytechnic University of New York, 2008.

[8] TS ETSI. 136101 V10. 5.0 Release 10. January 2012. LTE Evolved Universal Terrestrial Radio Access (E-UTRA): User Equipment (UE) radio transmission and reception.

[9] Kan Zheng, Fanglong Hu, Wenbo Wang, Wei Xiang, and Mischa Dohler. Radio resource allocation in LTE-advanced cellular networks with M2M communications. Communications Magazine, IEEE, 50(7):184-192, 2012.

[10] Roke Manor. LTE MAC Scheduler \& Radio Resource Scheduling. http://www.docstoc.com/docs/122980686/LTE-MAC-Schedulerand-Radio-Resource-Scheduling.

[11] Spark. Spark Cloud API. http://docs.spark.io/api/.

[12] SimuLTE LTE User Plane Simulation Model for INET OMNeT++ . https://github.com/inet-framework/simulte.

[13] G. Nardini A. Virdis, G. Stea. SimuLTE A Modular System-level Simulator for LTE/LTE-A Networks based on OMNeT++. 2014. 